\title{
Asma en el embarazo
}

\section{Asthma in pregnancy}

\author{
Clara Patricia García, MD.(1)
}

\section{Resumen}

El asma es una condición que ocurre también en el embarazo, y no es posible predecir si empeorará, se mantendrá igual o mejorará durante este. Es claro que requiere un control total de síntomas para la prevención de complicaciones, las cuales pueden aparecer frecuentemente durante la gestación, dada la fisiología que se modifica durante el embarazo. Por tanto, es crucial conocer los cambios fisiológicos, las pruebas diagnósticas y los métodos para evaluar el control de la enfermedad y estar al tanto de los riesgos de tratar y no tratar a las asmáticas durante el embarazo.

En esta revisión se abordan estos temas para el conocimiento de todos los llamados a manejar este grupo de pacientes.

Palabras clave: asma, exacerbaciones, fisiología en el embarazo, tratamiento, embarazo.

\begin{abstract}
Asthma is a condition that can appear during pregnancy. It is impossible to predict whether asthma will worsen, remain the same, or improve during pregnancy. It is clear that total control of symptoms is necessary to prevent complications, which can often appear during pregnancy due to gestational physiological changes. It is important to be acquainted with the physiological changes, the diagnostic tests, and the methods for assessing the control of the disease. It is likewise important to know the risks associated with treating and not treating women with asthma during pregnancy.
\end{abstract}

This review covers these subjects in order to increase the knowledgeability of all healthcare professionals who manage this group of patients.

Keywords: asthma, exacerbations, physiology in pregnancy, treatment, pregnancy.
${ }^{(1)}$ Internista, Neumóloga, Hospital Pablo
Tobón Uribe, Neumóloga Fundación An-
tioqueña de Infectología, Laboratorio de
Función Pulmonar. Medellín, Colombia.
Docente Honorario Universidad Tecnoló-
gica de Pereira, Colombia.
Correspondencia: Clara Patricia García,
correo electrónico: patogar@hotmail.com
Recibido: $27 / 05 / 15$. Aceptado: $07 / 06 / 15$. 


\section{Introducción}

El asma en una enfermedad respiratoria común, que acarrea una gran carga social y económica. Las embarazadas no son la excepción; se considera que el asma en la enfermedad respiratoria que más se reporta en ese estado. No obstante, se desconoce su prevalencia en ese contexto en Colombia, pero en el mundo se estima que está entre el 3 y el 8\% (1).

Los efectos del embarazo en el asma son variables. Aún se menciona la "hipótesis del tercio" la cual se traduce en un tercio que mejora, uno que empeora y uno que permanece igual que antes del embarazo. Así mismo, se han descrito algunas tendencias en el comportamiento del asma y su relación con el embarazo; la mayoría de autores consideran que la severidad del asma antes del embarazo se relaciona con la severidad durante el mismo (2).

Se cree que el asma es usualmente menos severa en las últimas cuatro semanas y en mujeres cuya enfermedad mejora con el embarazo; dicha mejoría será progresiva mientras trascurran los meses gestacionales. Una teoría que pudiera explicar este fenómeno es que en la medida que progresa el embarazo, la progesterona contribuye a la broncodilatación inducida por AMP cíclico. En aquellas mujeres en quienes empeora el control, esto es más pronunciado entre las semanas 29 a la 36 pero los síntomas usualmente son infrecuentes durante el trabajo de parto o el nacimiento, y el comportamiento del asma en embarazos subsecuentes será similar al previo (2).

Un marcador novedoso en asma y embarazo es la proteína de choque térmico 70 (HSP70), la cual es un marcador de inmunotolerancia reducido en pacientes embarazadas sanas comparadas con embarazadas con asma, pero aún no se conoce su relación con inflamación o desenlaces perinatales (3).

\section{Cambios fisiológicos durante el embarazo}

Para comprender un poco la relación entre asma y embarazo es importante repasar algunos conceptos sobre los cambios fisiológicos respiratorios en el embarazo. La mecánica de la vía aérea no cambia durante el embarazo. La capacidad vital forzada (CVF), el volumen espiratorio en el primer segundo $\left(\mathrm{VEF}_{1}\right)$ y la relación $\mathrm{VEF}_{1} / \mathrm{CVF}$ y pico espiratorio no cambian o están levemente aumentados. Por esto, una espirometría se interpreta igual en pacientes embarazadas y el diagnóstico se establece de la misma manera.

Los cambios fisiológicos durante el embarazo sí alteran otras variables. El incremento en los estrógenos ocasiona edema de la mucosa de la vía aérea e hipervascularización. A medida que la altura uterina aumenta, el diafragma se desplaza en forma cefálica y se eleva un promedio de 4 a $5 \mathrm{~cm}$, lo cual reduce la capacidad funcional residual. Los volúmenes pulmonares se modifican como se ve en la tabla 1. El consumo de oxígeno se eleva un promedio de $20 \%$ al incrementarse la tasa metabólica materna en un $15 \%$, y para compensar aumentos en la demanda, la ventilación minuto se aumenta un $40-50 \%$, pero a expensas de subir el volumen corriente y no la frecuencia respiratoria. Estos cambios se dan por el aumento de la progesterona, la cual estimula el centro respiratorio (4).

Tabla 1. Cambios en volúmenes pulmonares.

\begin{tabular}{|ll|}
\hline \multicolumn{1}{|c|}{ Volumen o capacidad pulmonar } & \multicolumn{1}{c|}{ Cambio durante el embarazo } \\
Capacidad vital & No se modifica \\
Capacidad pulmonar total & No se modifica excepto en el tercer trimestre donde disminuye levemente \\
Volumen residual & Disminuye $(20 \%-25 \%$ o $200-300 \mathrm{ml})$ \\
Capacidad funcional residual & Disminuye $(17 \%-20 \%$ o $300-500 \mathrm{ml})$ \\
Volumen de reserva espiratoria & Disminuye $(5 \%-15 \%$ o $100-300 \mathrm{ml})$ \\
\hline
\end{tabular}


Los gases arteriales se deben interpretar de diferente manera ya que la $\mathrm{PaCO}_{2}$ es menor (28 a $30 \mathrm{~mm}$ $\mathrm{Hg}$ a nivel del mar), mientras que la presión arterial de oxígeno es mayor $\left(\mathrm{PaO}_{2}\right.$ alrededor de 106 a 110 $\mathrm{mm} \mathrm{Hg}$ a nivel del mar) con un $\mathrm{pH}$ ligeramente alcalino $(7,40-7,45)$. En compensación, el bicarbonato aumenta su eliminación renal, lo que conlleva poliuria en los primeros meses de embarazo. Conviene tener presente que la presión arterial en la vena umbilical es menor que en las venas placentarias y por ello cualquier grado de hipoxemia materna se traduce en un descenso rápido en el contenido de oxígeno que le llega al feto. Si la hipoxemia es crónica se produce retraso del crecimiento uterino y bajo peso al nacer. En cualquier exacerbación, se necesario tener en cuenta los cambios en los gases, pues cualquier $\mathrm{CO}_{2}$ normal para un estado no gravídico, en el embarazo es criterio de fatiga ventilatoria (4).

En relación con las pruebas de broncoprovocación durante el embarazo, existe muy poca información pues la mayoría de los obstetras y otros especialistas no las solicitan durante ese estado (1).

\section{Afectación por asma en embarazadas y recién nacidos}

Muchos estudios relacionan asma y aumento de mortalidad perinatal, aborto espontáneo, hiperémesis, sangrado vaginal, preeclampsia, mortalidad neonatal, prematurez, enfermedad hipertensiva y bajo peso al nacer. Sin embargo, no todos los estudios son consistentes y tienen variabilidad en cuanto al tamaño y la selección de las muestras.

El estudio más grande elaborado es de 281.019 embarazos de los cuales 37.585 tenían asma. De igual forma, los embarazos de las asmáticas se relacionan con más abortos (OR 1,10; IC95\% 1,061,13 ), hemorragia preparto (OR 1,20; IC95\% 1,081,34), hemorragia postparto (OR 1,38; IC95\% 1,21-1,57), anemia (OR 1,06; IC95\% 1;01-1,12) y necesidad de cesárea (OR 1;11; IC95\% 1,07-1,16); no obstante, en estudios de cesáreas la mayoría eran electivas y no relacionadas con necesidad urgente de las mismas, razón por la cual ese dato debe interpretarse con cautela $(1,2)$.
Otro estudio de 36.985 pacientes con asma demostró un $20 \%$ de aumento de mortalidad perinatal, preeclampsia, parto pretérmino y de recién nacidos de bajo peso al nacer comparado con no asmáticas. Sin embargo, en estudios donde el asma se manejó de manera adecuada y activa no se demostró aumento de complicaciones perinatales. Así mismo, los estudios que han demostrado incremento en el retraso del crecimiento intrauterino o bajo peso al nacer son retrospectivos. En ninguna de las cohortes prospectivas se ha demostrado esto pero son estudios de pocos pacientes. Lo que sí es claro es que si las mujeres no venían usando esteroides inhalados si tenían más riesgo de este evento $(1,2)$.

En relación con la preeclampsia, la severidad del asma antes del embarazo sí es un factor asociado, en tanto que no se ha encontrado relación con la diabetes gestacional $(3,4)$.

La etiología de la mayoría de estas comorbilidades es conexa al no uso o abandono de uso de los esteroides inhalados así como de otros medicamentos, lo cual es frecuente dado que las mujeres renuncian a los medicamentos sin autorización de su médico al saber de su estado (5).

Otro factor por considerar es el psicológico. Se ha demostrado que el estrés y la ansiedad exacerban el asma en no embarazadas mientras que en incrementan en las embarazadas. Se ha demostrado, de igual forma, que hay un $52 \%$ de riesgo de sufrir depresión y se agrava cuando tienen una exacerbación durante el embarazo, lo cual contribuye al mal control de esta en dicho período (2).

\section{Crisis de asma}

Las exacerbaciones del asma en el embarazo suceden en un $20-36 \%$ de los casos, de los cuales un $5,8 \%$ requiere hospitalización. Aunque pueden aparecer en cualquier momento del embarazo, son más comunes al final del segundo trimestre. Así mismo, a mayor severidad del asma, más probabilidad de exacerbaciones durante el embarazo. En el estudio de Murphy et al. se reportaron exacerbaciones en un $8 \%$ de las pacientes con asma leve, en $47 \%$ con asma moderada y en $65 \%$ con asma severa $(6,7)$.

Revista Colombiana de Neumología Vol 27 No 3 | 2015 
De otro lado, se cree que el reflujo gastro-esofágico, la sinusitis, la obesidad, el mal control prenatal y especialmente la falta de uso de corticosteroides inhalados contribuyen al mal control de la enfermedad. En efecto, un tercio de las pacientes que tienen exacerbaciones o crisis severa no venía utilizando el esteroide inhalado antes del evento. Adicionalmente, hay estudios que demuestran una mayor tasa de ingresos a urgencias y consulta en pacientes no adherentes al esteroide inhalado (8).

De igual manera, las infecciones respiratorias virales están implicadas en las crisis asmáticas severas. Se sabe que la inmunidad celular se modifica en el embarazo pues no hay cambios humorales, disminución de la producción de anticuerpos en respuesta a antígenos solubles, menos células citotóxicas, menor cantidad de linfocitos $\mathrm{T}$ y disminución de la actividad de los NK (natural killers), lo cual aumenta la susceptibilidad a virus y hongos. Se ha visto, igualmente, menos síntomas de asma en mujeres vacunadas contra virus de influenza que en aquellas sin inmunización $(2,9)$.

\section{Tratamiento del asma durante el embarazo}

La Administración de Drogas y Alimentos (FDA, su sigla en inglés), ha determinado categorías para describir si una medicación ocasiona un efecto adverso durante el embarazo. Estas se elaboran con base en resultados de estudios en animales, información de estudios en humanos y consideraciones sobre riesgo/beneficio (tabla 2).

Las medicaciones de categoría $\mathrm{N}$ no ha sido clasificadas por la FDA.

Hasta el momento no hay ninguna medicación para asma o alergia en categoría A. Por tanto, es posible elegir medicamentos $\mathrm{B}$ y C pero las categorías D y X están contraindicadas en el embarazo.

Los dos objetivos primarios en el asma son la prevención de un evento de exacerbación lo cual puede disminuir el riesgo de hipoxia aguda fetal, hipocapnia, alcalosis y deshidratación que puede afectar al bebe en desarrollo; y la optimización de la función pulmonar en la madre para prevenir hipoxemia crónica, crisis de asma y mejorar síntomas.

Para lograr estos objetivos una parte involucra la monitorización de la función pulmonar, en particular en este grupo de pacientes en quienes puede ser difícil diferenciar la disnea fisiológica del embarazo de una crisis. Para esto se propone el control con medidor de pico flujo o medición espirométrica del $\mathrm{VEF}_{1}$; sin embargo, el espirómetro está poco disponible en nuestro medio en

Tabla 2. Categorías de seguridad de la FDA para medicamentos empleados en asma durante el embarazo.

\begin{tabular}{|c|l|}
\hline Categoría de la FDA & \multicolumn{1}{c|}{ Característica de la categoría } \\
A & $\begin{array}{l}\text { Estudios bien controlados no han demostrado riesgo al feto durante el primer trimestre del } \\
\text { embarazo (no hay evidencia de riesgo en otros trimestres). }\end{array}$ \\
B & $\begin{array}{l}\text { Los estudios en animales han fracasado en demostrar riesgo en el feto pero no hay estudios } \\
\text { bien controlados en mujeres embarazadas. }\end{array}$ \\
C & $\begin{array}{l}\text { Los estudios en animales han mostrado efecto adverso en el feto y no hay estudios adecuados } \\
\text { bien controlados en humanos pero los beneficios pueden ser superiores a los riesgos } \\
\text { potenciales. }\end{array}$ \\
D & $\begin{array}{l}\text { Hay evidencia positiva de riesgo en el feto humano por eventos adversos en datos de estudios } \\
\text { experimentales en humanos pero los beneficios pueden requerir su formulación a pesar de } \\
\text { riesgos potenciales. }\end{array}$ \\
X & $\begin{array}{l}\text { Los estudios en animales y en humanos han demostrado anomalías fetales y hay evidencia } \\
\text { positiva de riesgo en feto humano basado en estudios experimentales o promocionales. Los } \\
\text { riesgos de la medicación claramente sobrepasan posibles beneficios. }\end{array}$ \\
\end{tabular}


los consultorios médicos. El pico espiratorio flujo durante el embarazo oscila entre 380 y $550 \mathrm{~L} / \mathrm{min}$ y es deber del médico tratante educar a la paciente en cuanto a su utilización de manera que sepa cuál es su mejor pico flujo histórico $(100 \%)$ y de ahí establezca límites para modificar conductas. Cuando el pico flujo esté entre el $80-100 \%$ de su mejor valor, está controlado; si está entre 50 y $80 \%$ deberá consultar para ajustar la terapia y es menor del $50 \%$ deberá asistir por urgencias si no hay cita prioritaria disponible.

En cuanto a herramientas de control por síntomas en embarazadas, el ACT (Asthma Control Test) es una herramienta disponible desde el 2004. Originalmente se compone de cinco preguntas con relación a la frecuencia de síntomas de asma e impacto funcional de la enfermedad y necesidad de rescates en las últimas cuatro semanas. El puntaje oscila entre 5 (pobre control) y 25 (control total). Por encima de 20 se considera buen control y por debajo de 15 mal control; ya se tiene un estudio de validez en el embarazo en un grupo en Brasil $(10,11)$.

Sin embargo, recientemente otro grupo propuso una modificación en la pregunta 2 referente a la disnea para discriminar mejor de disnea fisiológica del embarazo con una validez adecuada, poder diferenciar entre control bueno y pobre y hacer seguimiento, incluso telefónico, de manera que sea más objetiva la evaluación del control de la enfermedad en este grupo (12).

En cuanto al manejo no farmacológico es importante el cese del tabaquismo, educación en asma con énfasis en la seguridad de las medicaciones, el control del estrés y la ansiedad que agravan la enfermedad y el estímulo al buen control de ésta para minimizar complicaciones durante la gestación.

El manejo farmacológico incluye medicamentos que se describen a continuación.

Beta dos agonistas inhalados: la mayoría de estudios reportan seguridad durante el embarazo. La experiencia clínica es superior con drogas antiguas como beta dos de corta acción (SABA) que con los de la larga acción (BALA). Son medicamentos que proveen alivio rápido de los síntomas. Aunque existen reportes de algunos casos de gastrosquisis en pacientes que los usaron desde el primer trimestre, se cree que estaban mas relacionados con el asma no controlada que con la droga por si misma (13).

Hay menor evidencia del uso de BALA (beta dos de larga acción) en especial formoterol y salmeterol. En los estudios animales no hay aumento de anomalías congénitas; por tanto, si la paciente lo requería antes del embarazo para mantener el control de su enfermedad, no debe ser suspendido. Sin embargo, se recomienda su uso con corticosteroide inhalado por las mismas razones cardiovasculares descritas en no embarazadas.

Los corticosteroides orales o sistémicos son la piedra angular para al control de las pacientes asmáticas en estado de embarazo. Algunos estudios reportan alarmas respecto al riesgo de algunas malformaciones fetales. En estudios animales, el uso de altas dosis sistémicas puede conllevar a paladar hendido. El cierre del paladar usualmente se presenta en la semana 12, por lo cual algunos sugieren que no se usen sistémicos en el primer trimestre. En un metaanálisis que incluye seis estudios de cohorte y cuatro de casos y controles del uso de esteroides sistémicos en el primer trimestre, no halló una asociación clara con malformaciones congénitas totales $(\mathrm{OR}=1,45 ; \mathrm{IC} 95 \% 0,80-2,60)$, si bien en los casos y controles sí encontraron más labio y paladar hendido $(\mathrm{OR}=3,35$; IC95\% 1,97$5,69)(14,15)$.

En cuanto a parto pretérmino y recién nacido de bajo peso al nacer hay una cohorte prospectiva de 2.123 embarazadas con asma, en la que el uso de esteroide oral, sin ser específico el tiempo de duración de su uso, se asocia con parto prematuro $(\mathrm{OR}=1,54$; IC 95\% 1,02-2,33) y bajo peso al nacer $(\mathrm{OR}=1,80$; IC95\% 1,13-2,88) (16).

Otros efectos descritos son preeclampisa, diabetes gestacional e hipertensión materna por el uso de esteroides orales. 
Por el contrario, los estudios con el uso de corticosteroide inhalado han demostrado seguridad, especialmente con budesonida, la cual es la única en categoría B según la FDA. Sin embargo, también hay estudios de seguridad con beclometasona y otros con fluticasona en los que se indica riesgo de malformaciones mayores (OR de 1,1; IC 95\% 0,5-2,3). En otro estudio de 13.280 embarazos de pacientes asmáticas con dosis consideradas bajas o moderadas, se concluyó que esto no lleva a malformaciones pero las dosis de beclometasona mayores de $1.000 \mathrm{mcg}$ o sus equivalentes en el primer trimestre aumenta algunas malformaciones totales; sin embargo, nuevamente los autores concluyen que no pueden diferenciar si es un efecto por la medicación o por la severidad del asma por si misma. Sin embargo, estudios prospectivos de manejo con beclometasona después de una crisis versus no usarla, han demostrado que el uso disminuye los reingresos a urgencias, sin encontrarse efectos adversos, por lo cual se recomienda su formulación (17-20).

En relación al omalizumab se considera categoría $\mathrm{B}$ y se recomienda no usarlo a menos que se haga necesario por mal control. Los estudios fueron hechos en animales predominantemente, aunque hay reportes de casos de seguridad en alguna mujer que lo venía recibiendo antes de quedar en embarazo. $Y$ el estudio EXPECT que mostró seguridad pero con bajo número de pacientes y presencia de algunos efectos adversos similiares a los descritos para otras drogas como prematurez, embarazo pretérmino, bajo peso y algunas malformaciones menores en bajo número de pacientes $(21,22)$.

Con relación a las crisis de asma las recomendaciones generales son las siguientes:

1. Las dosis de esteroides sistémicos en crisis son iguales a las de mujeres no embarazadas.

2. No se recomienda el uso de aminofilina o teofilina.

3. El sulfato de magnesio puede ser útil en crisis severa como coadyuvante de esteroide intravenoso y beta dos inhalado, más si hay amenaza de parto prematuro o hipertensión.
4. Se recomienda el uso de beta dos agonistas y anticolinérgicos y esteroides inhalados de igual forma que en no embarazadas.

En las recomendaciones periparto es de mencionar que no hay contraindicación para el uso de oxitocina. De los análogos de prostaglandinas para dilatar el cérvix o control de hemorragia uterina el F2 alfa está completamente contraindicado pues se asocia a broncoconstricción; se puede usar análogo de prostaglandina $\mathrm{E}_{2}$ en gel o supositorio o el misoprostol que es análogo de la prostaglandina $\mathrm{E}_{1}$.

Para analgesia postparto no se recomienda morfina o meperidina pues pueden inducir broncoconstricción por liberación de histamina pero no se ha demostrado que suceda agudamente. El fentanil sí es seguro. El uso de anestesia epidural es ideal para reducir consumo de oxígeno y ventilación minuto en el trabajo de parto (1).

\section{Conclusión}

El asma durante el embarazo es la enfermedad respiratoria más prevalente y es vital el tratamiento adecuado a fin de evitar complicaciones maternas y fetales. La mayoría de métodos diagnósticos se interpretan igual durante el embarazo pero sí hay cambios fisiológicos que afectan de manera intrínseca algunos de estos parámetros, en particular los gases arteriales. Algunas pruebas como la metacolina no se realizan durante el embarazo, pero en general son las mismas que para la población no embarazada. En la actualidad, el ACT es el cuestionario validado durante la gestación para determinar el control; adicionalmente, siempre se debe estimular a llevar a la embarazada al control total. El manejo es similar al de las no embarazadas, en cuyo caso los esteroides inhalados siguen siendo la piedra angular en el manejo aunque se han descrito enfermedades congénitas ligadas a su uso; sin embargo, la mayoría de autores considera gran parte de los medicamentos, excepto la teofilina, seguros en el embarazo y sus efectos secundarios parecen estar más ligados a la severidad del asma que a estos por sí mismos.

\section{Conflictos de interés}

La autora declara no tener conflictos de interés. 


\section{Bibliografía}

1. Weinberger SE, Schatz M. Physiology and clinical course of asthma in pregnancy (internet). (place unknown). 2016. (updated 2016 Feb) Disponible en: http://www.uptodate.com/ home

2. Murphy VE, Gibson PG. Asthma in Pregnancy. Clin Chest Med. 2011;32:93-110.

3. Tamasi L, Bohacs A, Tamasi V, Stenczer B, Prohászka Z, Rigó $\mathrm{J} \mathrm{Jr}$, et al. Increased circulating heat shock protein 70 levels in pregnant asthmatics. Cell Stress Chaperones. 2010;15:295.

4. Hardy-Fairbanks AJ, Baker ER. Asthma in pregnancy: pathophysiology, diagnosis and management. Obstet Gynecol Clin N Am. 2010;37:159-72.

5. Schatz M, Leibman C. Inhaled corticosteroid use and outcomes in pregnancy. Ann Allergy Asthma Immunol. 2005;95:234.

6. Murphy VE, Gibson P, Talbot PI, et al. Severe asthma exacerbations during pregnancy. Obstet Gynecol. 2005;106:104654.

7. Stenius-Aarniala BS, Hedman J, Teramo KA. Acute asthma during pregnancy. Thorax. 1996;51:411.

8. Schatz M, Leibman C. Inhaled corticosteroid use and outcomes in pregnancy. Ann Allergy Asthma Immunol. 2005;95:234.

9. Graves C. Pneumonia in pregnancy. Obstet Gynecol. 2010;53( 2):329-36.

10. Nathan RA, Sorkness CA, Kosinski M, Schatz M, Li JT, Marcus P, et al. Development of the asthma control test: a survey for assessing asthma control. J Allergy Clin Immunol. 2004; $113: 59-65$

11. Monteiro M, Rizzo JA, de Melo Junior EF, Pires Lins ESL$\mathrm{ME}$, et al. Validation of the Asthma Control Test in pregnant asthmatic women. Respir Med. 2014;108:1589-93.

12. Palmsten $\mathrm{K}$, Schatz $\mathrm{M}$, Chan $\mathrm{PH}$, et al. Validation of the pregnancy asthma control test. J allergy Clin Immunol Pract. 2015;4(2):310-5.
13. Garne E, Hansen AV, Morris J, et al. Use of asthma medication during pregnancy and risk of specific congenital anomalies: A European case-malformed control study. J Allergy Clin Immunol. 2015;136:1496.

14. Eltonsy S, Forget A, Beauchesne MF, Blais L. Risk of congenital malformations for asthmatic pregnant women using a long-acting $\beta 2$-agonist and inhaled corticosteroid combination versus higher-dose inhaled corticosteroid monotherapy. J Allergy Clin Immunol. 2015;135:123.

15. Park-Wyllie L, Mazzotta P, Pastuszak A, et al. Birth defects after maternal exposure to corticosteroids: prospective cohort study and meta-analysis of epidemiological studies. Teratology. 2000;62:385.

16. Schatz M, Dombrowski MP, Wise R, et al. The relationship of asthma medication use to perinatal outcomes. J Allergy Clin Immunol. 2004;113:1040.

17. Källén B, Rydhstroem H, Aberg A. Congenital malformations after the use of inhaled budesonide in early pregnancy. Obstet Gynecol. 1999;93:392.

18. Charlton RA, Snowball JM, Neghtingale AL, Davis KJ. Prescribed for Asthma During Pregnancy: A UK Population-Based Cohort Study. J Allergy Clin Immunol Pract. 2015;3:772.

19. Blais L, Beauchesne MF, Lemière C, Elftouh N. High doses of inhaled corticosteroids during the first trimester of pregnancy and congenital malformations. J Allergy Clin Immunol. 2009;124:1229.

20. Wendel PJ, Ramin SM, Barnett-Hamm C, et al. Asthma treatment in pregnancy: a randomized controlled study. Am J Obstet Gynecol. 1996;175:150.

21. Hirashima J, Hojo M, Iikura M. A case of an asthma patient receiving omalizumab during pregnancy. Arerugi. 2012;61(11):1683-7.

22. Namazy J, Cabana MD, Scheuerle AE. The Xolair Pregnancy Registry (EXPECT): the safety of omalizumab use during pregnancy. J Allergy Clin Immunol. 2015;135(2):407-12. 